\title{
Experiencias y beneficios del uso de Arduino en un curso de programación de primer año
}

\author{
Miguel Tupac-Yupanqui ${ }^{1^{\star}}$, Cristian L. Vidal-Silva ${ }^{2^{*}}$, Aurora Sánchez-Ortiz ${ }^{2^{\star}}$, y Felipe Pereira ${ }^{3^{*}}$ \\ (1) EAP Ingeniería de Sistemas e Informática, Universidad Continental, Huancayo - Perú (correo-e: \\ mtupac@continental.edu.pe) \\ (2) Departamento de Administración, Facultad de Economía y Administración, Universidad Católica del Norte, Angamos \\ 0610, Antofagasta - Chile (correo-e: cristian.vidal@ucn.cl, asanchez@ucn.cl) \\ (3) Escuela de Educación, Universidad Viña del Mar, Viña del Mar - Chile (correo-e: fpereria@uvm.cl)
}

* Autor a quien debe ser dirigida la correspondencia

Recibido Mar. 17, 2021; Aceptado May. 24, 2021; Versión final Jun. 16, 2021, Publicado Dic. 2021

\begin{abstract}
Resumen
Este estudio presenta la experiencia del uso de Arduino y la plataforma Tinkercad para el desarrollo de competencias de programación en estudiantes de primer año en la Universidad Continental de Huancayo (Perú). El estudio es desarrollado en línea durante tiempos de la pandemia COVID-19 con uso de un simulador. El desarrollo de competencias de programación es un hito fundamental y esencial para estudiantes de carreras de ingeniería de sistemas, ciencias de la información, computación e informática. Para los estudiantes, resulta complejo trabajar con lenguajes de programación que requieren el cumplimiento de reglas tanto sintácticas y semánticas complejas. Los resultados demuestran un impacto positivo en el desarrollo de competencias de los estudiantes respecto a cursos previos, además de desarrollar competencias básicas del área de circuitos digitales y electrónica computacional. Se concluye que los estudiantes se sienten muy cómodos con el uso de Arduino y Tinkercad, aun cuando por la pandemia, los experimentos no fueron desarrollados de forma física.
\end{abstract}

Palabras clave: Arduino; Tinkercad; algoritmos; programación; competencias de los estudiantes

\section{Experiences and benefits of using Arduino in a first-year programming course}

\begin{abstract}
This study presents the experience of using Arduino and the platform Tinkercard for developing programming competencies in first-year students at the Continental University of Huancayo (Peru). The study is conducted online during the COVID-19 pandemic by using a simulator. The development of programming competencies is a fundamental and essential milestone for students enrolled in systems engineering, information science, computer science, and informatics degrees. For students, it is challenging to work with programming languages that require compliance with complex syntactic and semantic rules. The results show and demonstrate a positive impact on the development of students' skills when compared to previous courses. Development of basic skills is observed on digital circuits and computational electronics. It is concluded that students are highly comfortable with Arduino and Tinkercard, even though, due to the pandemic, the experiments are not performed physically.
\end{abstract}

Keywords: Arduino; Tinkercad; algorithms; programming; student competences 


\section{INTRODUCCIÓN}

Según el trabajo de Vidal et al (2015), un algoritmo representa un conjunto de pasos precisos, finitos e inequívocos para obtener una meta definida. En ciencias de la computación, programar o escribir un programa significa dar un conjunto de instrucciones a la computadora para su ejecución y entonces lograr un propósito definido; esto es, los programas escritos en lenguajes de programación representan algoritmos (Vidal et al., 2015). La programación puede resultar una experiencia placentera como escribir poesía o componer música. Estimular el desarrollo del pensamiento algorítmico en los niños representa los objetivos establecidos de los sistemas educativos indiferentes a los países europeos (Young, 2017; Malik y Coldwell-Neilson, 2017) y sudamericanos (Velasco-Ramírez, 2020) porque el desarrollar competencias algorítmicas permite desarrollar competencias de análisis, diseño y soluciones de problemas (Vidal et al., 2015). Sin embargo, tal como afirma (Troussas et al., 2020), el desarrollo de estas competencias resulta ser un problema de enseñanza aprendizaje tanto para profesores y estudiantes.

El desarrollo de competencias algorítmicas y de lenguajes de programación resulta esencial en la formación de profesionales y técnicos de las áreas de sistemas y ciencias de la información (González-Guerra y LealFlores, 2020). Con este propósito, cursos de introducción a los algoritmos y programación comúnmente están en los primeros semestres de dichas carreras. Por ejemplo, la malla curricular de la carrera Ingeniería de Sistemas e Informática de la Universidad Continental - Perú (UCON-Malla, 2021) presenta el curso de fundamentos de programación en el segundo semestre. Así mismo, la nueva malla curricular de Ingeniería en Información y Control de Gestión de la Universidad Católica del Norte - Chile considera un curso de programación durante el primer semestre del primero año académico (IICG-Malla, 2021).

Tal como señalan Qian y Lehman (2017), la ciencia de la programación, además de competencias del área de resolución de problemas, también incluye conceptos abstractos fuertemente conectado con la mecánica de programar la cual está estrechamente ligada a la estructura sintáctica y semántica del lenguaje de programación utilizado. Vidal et al. (2015) argumentan que el desarrollo de competencias de programación utilizando lenguajes de programación textuales requiere una abstracción de alto nivel junto con habilidades de enfoque principal y razonamiento algorítmico. Sin embargo, los estudiantes que usan habitualmente lenguajes de programación textuales pasan tiempo entendiendo y respetando las reglas de sintaxis en lugar de comprender y desarrollar competencias algorítmicas y resolver el problema. Más aún, las nuevas generaciones de estudiantes utilizan más dispositivos visuales que las generaciones previas (Madigan et al., 2020), y los profesores necesitan herramientas más adecuadas para enseñar (González-Guerra y Leal-Flores, 2020).

Este trabajo presenta experiencias académicas con el uso de sistemas Arduino para el desarrollo de competencias de programación en estudiantes de primer año de Universidad. Según los resultados obtenidos, por el alto nivel de interacción con el ambiente que se logra con sistemas Arduino, el desarrollo de competencias algorítmicas y de programación son altamente factibles, además de desarrollar competencias básicas de electricidad y electrónica.

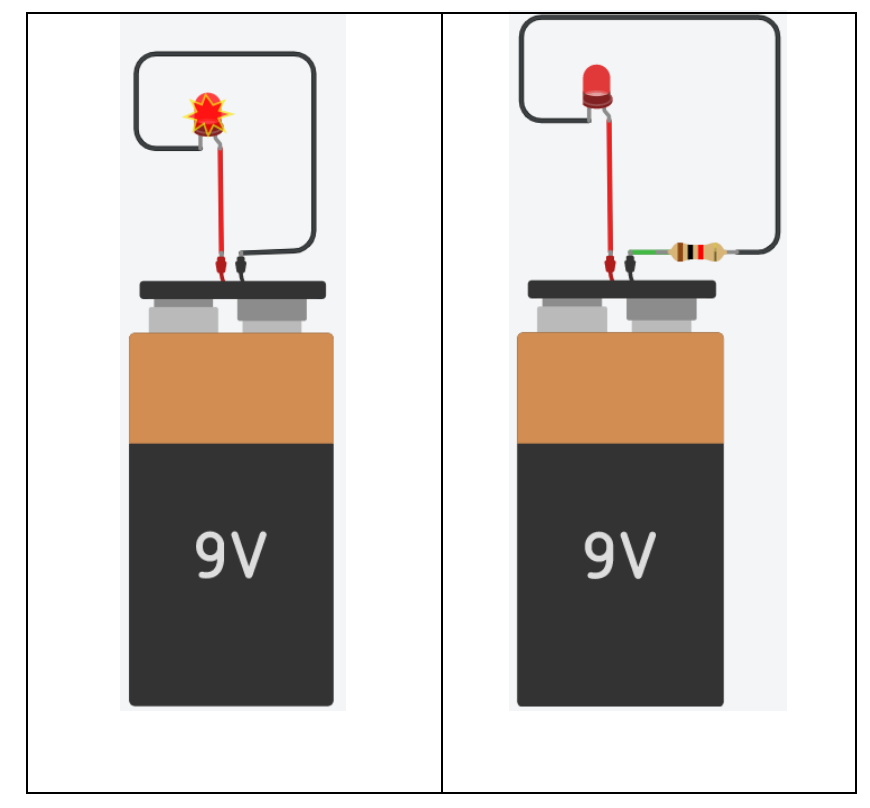

Fig.1. Ejemplo Hola Mundo en simulador Arduino Tinkercad 


\section{OTROS ANTECEDENTES}

A continuación, se entregan detalles de la plataforma de hardware libre Arduino, y del simulador Tinkercad para el diseño y prueba de sistemas Arduino. Aun cuando Tinkercad no considera todos los componentes posibles de integrar en un sistema Arduino, esta plataforma si integra componentes hardware que son base y posibles de adquirir para la realización de experimentos reales.

\section{Arduino}

Gracias a los avances tecnológicos tanto en hardware como en software, en la búsqueda de una mayor integración de los mismos, cada día es más viable el desarrollo de soluciones integradas de hardware y software para estudiantes y profesionales de dichas áreas. Por ejemplo, tal y como se describe en el trabajo de Vidal y Pavesi (2005), una solución integrada de hardware y software tradicional requiere fragmentar su diseño en hardware y software sin perder de vista su integración. Aun cuando los sistemas de computación de escritorio reflejan grandes avances tanto en velocidad de cómputo y capacidad de memoria, estos son sistemas que involucran responsabilidades adicionales tanto de sistema operativo como de usuario, además de la responsabilidad de la existencia de señales externas para su recepción y procesamiento. Es decir, existe un delta de tiempo entre captura y procesamiento de la señal que para señales de alta variabilidad, según Teorema de Shanon y Nyquists (Vidal y Pavesi, 2005), los sistemas computacionales tradicionales puede resultar poco práctico para trabajar con sistemas de recepción y procesamiento de señales.

La tecnología Arduino resulta en un sistema de computación con la integración directa de las áreas de hardware y software para el diseño e implementación de soluciones. Arduino hoy representa una compañía de código y hardware abierto para facilitar el acceso y uso conjunto de la electrónica y computación para el desarrollo de sistemas (Vidal et al., 2019). Al igual que una computadora convencional, una placa Arduino puede realizar una multitud de funciones, y requiere de entradas y/o salidas para un mayor valor de su uso. En la práctica, ya existen diferentes placas Arduino disponibles tales como Arduino Uno R3, Arduino Leonardo y Arduino Mega, donde los dos últimos representan una evolución desde Arduino Uno R3 en términos de mejoras e inclusión de nuevas características hardware (Sirkin et al., 2017). Por ejemplo, una versión de Arduino Mega permite la interacción con teléfonos móviles Android.

A continuación, se describen detalles de componentes esenciales de Arduino Uno R3 usado en el desarrollo de los proyectos base de este trabajo: microcontrolador ATmega328; voltaje de entrada 7-12V; 14 pines digitales de I/O, con 6 salidas PWM para la generación de señales analógicas; 6 entradas análogas; 32KB de memoria Flash; Reloj de $16 \mathrm{MHz}$ de velocidad. La figura 2 muestra y detalla los componentes de Arduino Uno R3. Dadas las entradas y salidas de una placa Arduino, es factible la interacción con diferentes componentes y dispositivos de electrónica con el mismo nivel de voltaje. Como ejemplos de uso y aplicación de Arduino, Vidal et al. (2019) presenta ejemplos para la adquisición de señales y la acción de dispositivo para posiblemente afectar el valor de estas señales.

El diseño de soluciones electrónicas parece una tarea compleja por los dispositivos físicos comprometidos en la implementación del circuito electrónico final, un riesgo que desaparece con el uso de simuladores. Arduino es una plataforma electrónica de código abierto para desarrollar proyectos interactivos de computación y electrónica (Sirkin et al., 2017). El enfoque de programación de Arduino es sintáctica y semánticamente análogo a C / C ++ (Perenc et al., 2019), además de la precaución para trabajar en dispositivos físicoelectrónicos; ambas tareas realmente complejas para los estudiantes novatos en programación. Los circuitos de la plataforma web Tinkercad (2021) parecen buscar resolver problemas de programación y diseño de sistemas Arduino: Tinkercad unifica un lenguaje de programación estructurado en bloques semejante a Scratch (Resnick et al., 2009; Vidal et al., 2019), con el uso de componentes electrónicos compatibles con Arduino para diseñar y programar soluciones informáticas. La figura 1 muestra un ejemplo básico $u$ hola mundo en electrónica para conectar un led a una batería de 9 voltios y así encenderlo.

\section{Circuitos Tinkercad}

Tinkercad (2021) es una plataforma web gratuita y fácil de usar para el diseño 3D, la electrónica y la codificación en el desarrollo de soluciones informáticas electrónicas que utilizan Arduino. Arduino se creó durante 2005 en Italia buscando integrar fácilmente la informática y la electrónica para estudiantes en contextos de hardware abierto y de bajo costo (Lineros et al., 2018). Hoy en día, existe un conjunto variado de artículos de hardware y software de bajo costo compatibles con Arduino en el mercado. Por ejemplo, existen pantallas de cristal líquido, sensores y plataformas de desarrollo web integradas como Tinkercad. 


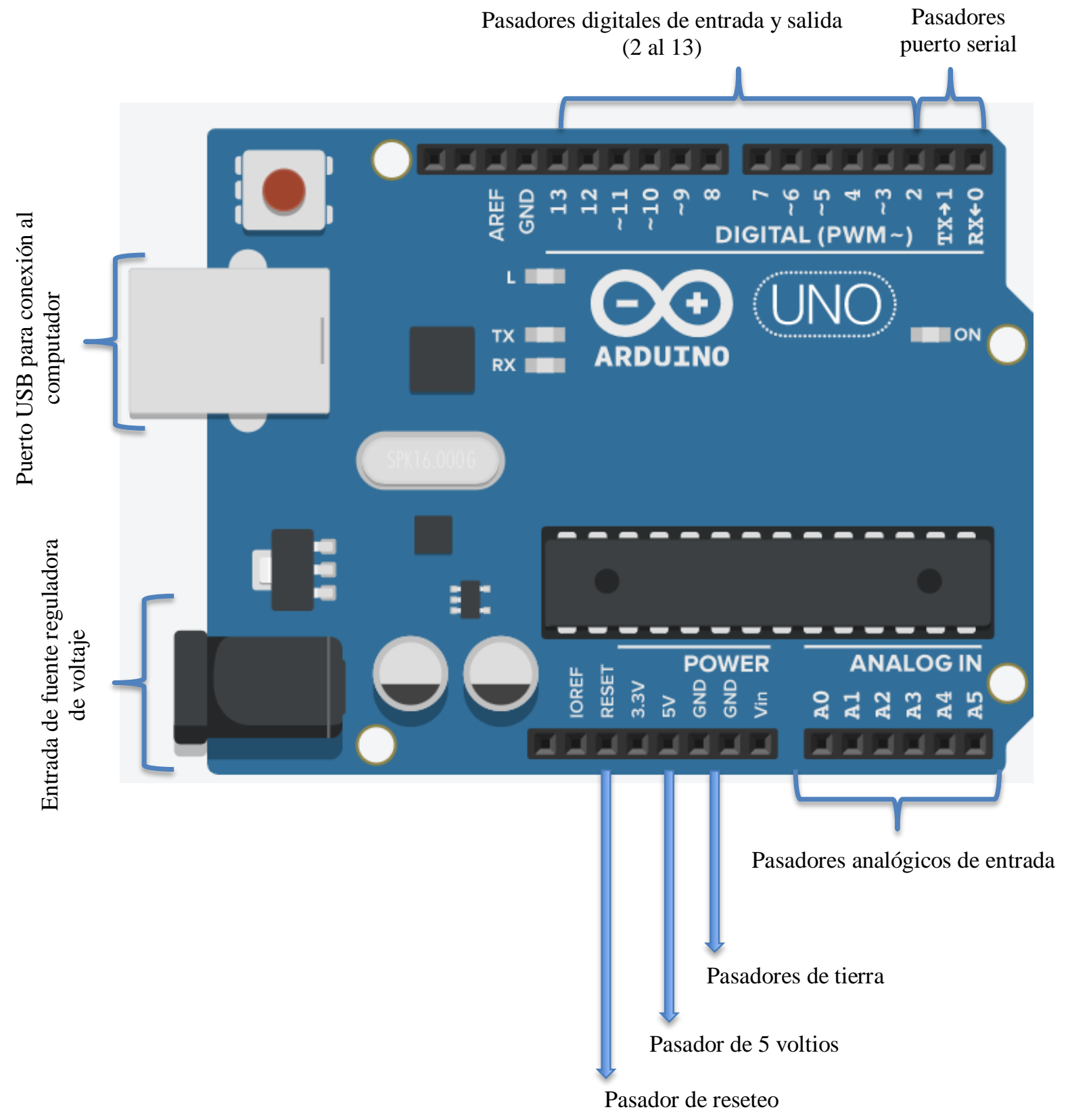

Fig.2. Entradas / salidas de Arduino Uno

Tinkercad respalda y motiva el desarrollo de soluciones con Arduino. Tinkercad considera un área de diseño para los componentes de hardware en la solución, una clasificación de diferentes componentes de hardware capaces de agregar y un área de código para escribir el programa. La figura 3 muestra una solución de hardware diseñada en Tinkercad para encender un led rojo, donde Arduino ahora está a cargo de enviar las señales positivas y de tierra. Agregar programación a esta solución permite encender cíclicamente el led por un tiempo t1 y apagarlo por otro tiempo t2. Programación Las soluciones Arduino requieren conocimientos avanzados de programación para la nueva sintaxis y semántica de su lenguaje de programación similar a C / $\mathrm{C}++$. Sin embargo, Tinkercad permite la solución de programación mediante un lenguaje estructurado en bloques como Scratch. La figura 4 muestra la clasificación coloreada de los bloques con respecto a su propósito Salida (azul), Entrada (púrpura), Notación (gris), Control de flujo (amarillo), Matemáticas (verde) y Variables (rosa) junto con la sección para la definición de variables. La figura 4 además muestra el código estructurado en forma de bloques para encender y apagar el led en los tiempos t1 y t2, respectivamente. La figura 5 muestra el código del lenguaje de programación Arduino cuya sintaxis y semántica es similar a lenguaje de programación C. 


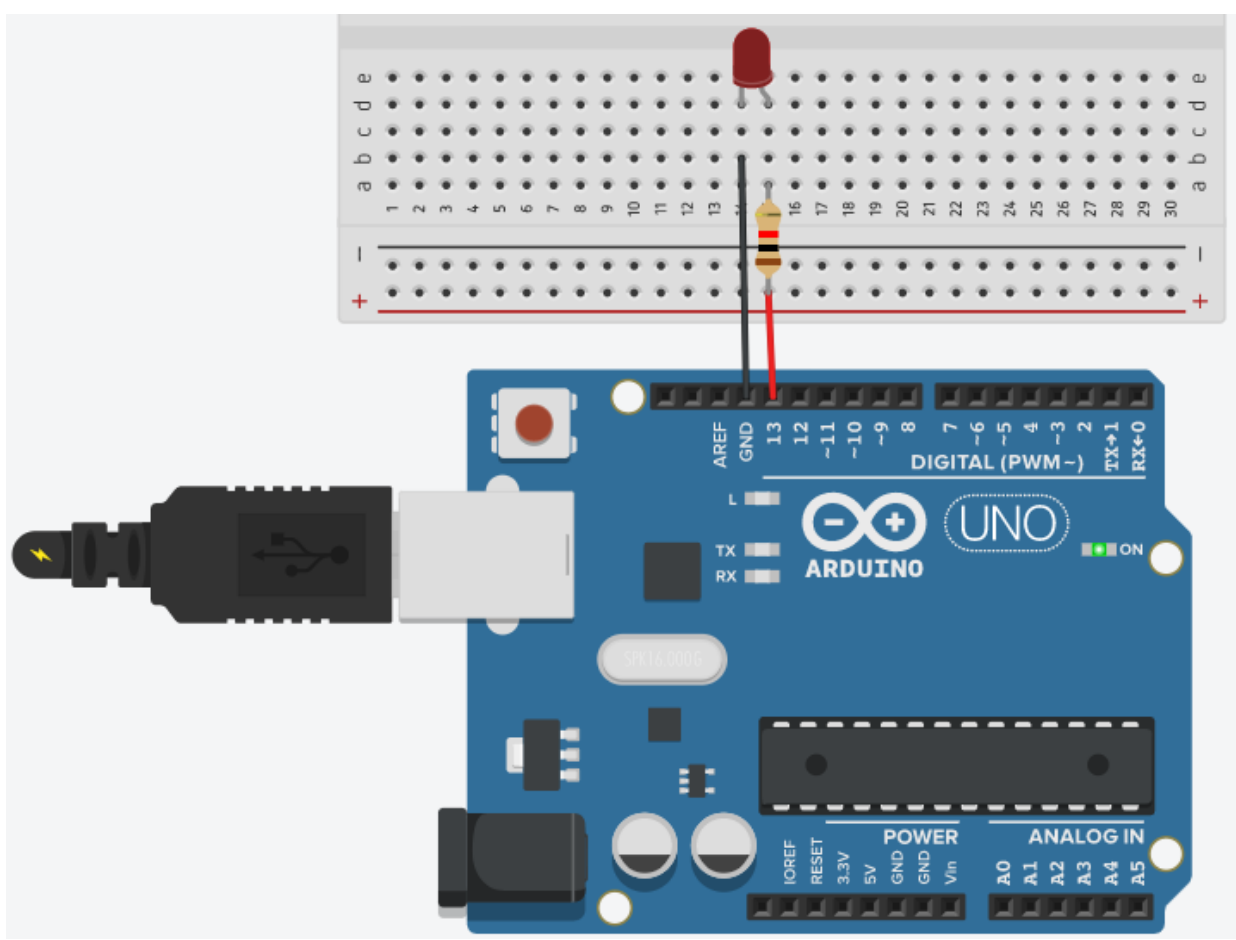

Fig. 3. Ejemplo Tinkercad Arduino para encender un led

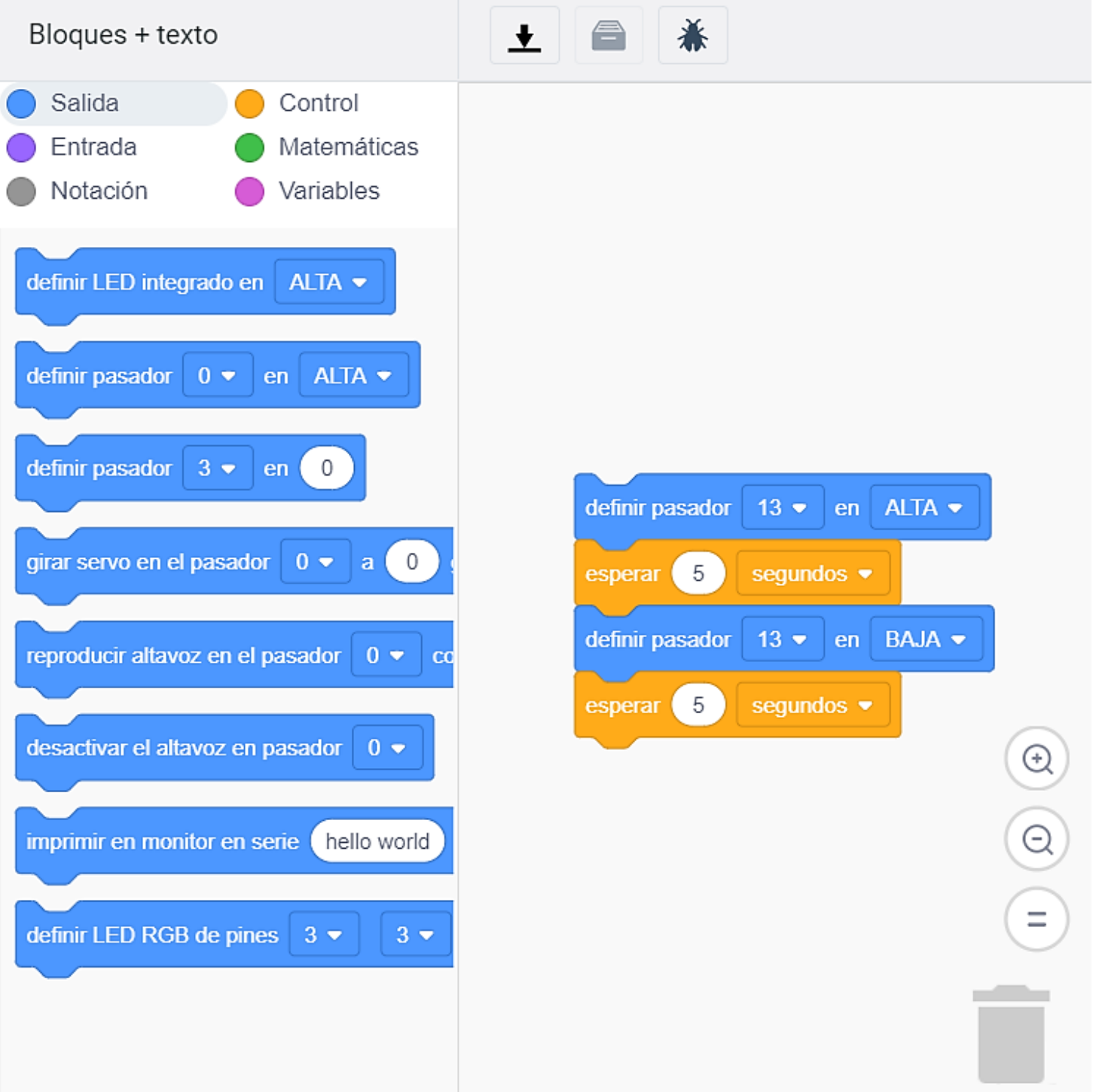

Fig. 4. Código en bloques ejemplo en Tinkercad 


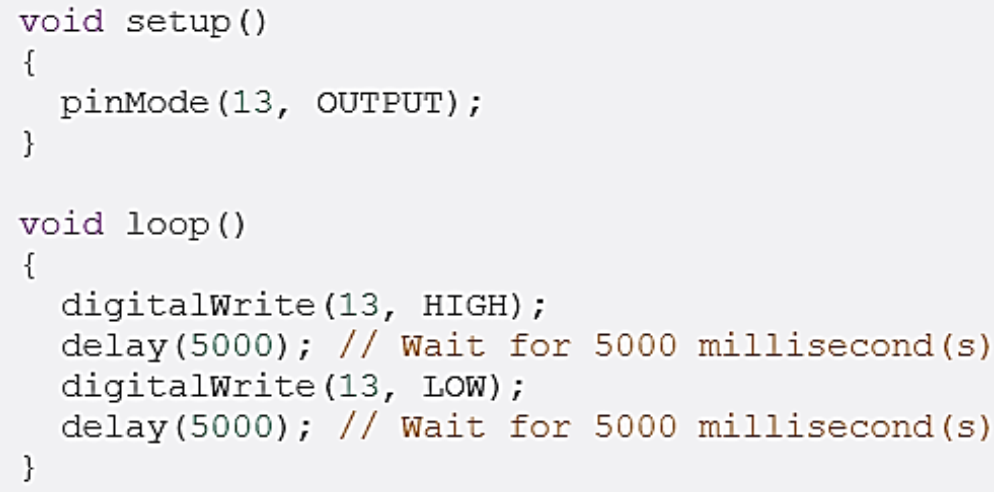

Fig. 5. Código Arduino ejemplo en Tinkercad

Tinkercad también permite generar el código C / C ++ para la solución Arduino a partir de una solución de código estructurado en bloques que representa una propiedad excelente. Por lo tanto, para desarrollar una solución simple, Tinkercad es una increíble plataforma web para comenzar a aprender soluciones informáticas electrónicas basadas en Arduino.

\section{METODOLOGÍA}

Según programas de estudios de módulos o asignaturas de programación, en general, se definen las siguientes competencias: 1. Capacidad para definir una solución algorítmica a un problema computacional (Juškevičienè, 2020), 2. Capacidad de escribir solución computacional en un lenguaje de programación, 3. Capacidad de trabajo en equipo (Chorfi, 2020). A continuación, se presentan objetivos de aprendizaje para el desarrollo de dichas competencias: 1.1. Dominio teórico / práctico de operaciones de entrada / salida en soluciones algorítmicas, 1.2. Dominio teórico / práctico de variables en soluciones algorítmicas, 1.3. Dominio teórico / práctico de estructuras de control en soluciones algorítmicas, 1.4. Dominio teórico / práctico de estructuras repetitivas en soluciones algorítmicas, 1.5. Dominio teórico / práctico de subrutinas y pensamiento modular en soluciones algorítmicas; 2.1. Representar soluciones algorítmicas en un lenguaje de programación, 2.2. Medir factibilidad y eficiencia práctica de soluciones de programación; y 3.1. Organización de responsabilidades y tareas en equipo, 3.2. Autoevaluación y coevaluación de trabajo.

La carrera de Ingeniería de Sistemas de la Universidad Continental, para la consecución de las competencias y objetivos de aprendizaje de curso de introducción a la programación, durante 2020 se considera el uso de Arduino y la plataforma Tinkercad como apoyo desde la octava semana del curso. Anterior a eso, se utilizan las herramientas PSelnt (2021) y Raptor (Hooshyar et al., 2014) durante las primeras siete semanas del curso. El curso se dicta durante ambos semestres del año académico, y durante 2020, cuando el mundo vive tiempos de pandemia, las sesiones debieron ser en línea.

Es importante destacar que, para lograr las competencias de programación con Arduino y Tinkercad, durante la primera sesión se inicia con los ejemplos de las figuras 1, 2 y 3 . Entre las sesiones dos y cinco inclusive, se resalta la estructura básica de soluciones Arduino (funciones de configuración setup y de ciclo loop), mediante ejemplos algorítmicos en lenguaje de programación por bloques. Nótese que aun cuando no hay directamente una pantalla para visualizar salidas de un sistema Arduino, tanto la simulación Tinkercad como la potencial experiencia con circuitos físicos permiten lograr el efecto visual positivo y necesario para con los estudiantes.

\section{RESULTADOS}

La tabla 1 muestra resume estadísticas de las calificaciones finales de los estudiantes para los años académicos 2019 y 2020 en cada uno de sus semestres. Es importante destacar que durante todo el año 2019 se utilizaba un lenguaje de programación tradicional (C y Java en este caso), mientras que durante 2020 y para cada uno de sus semestres académicos, se utilizaba Arduino y Tinkercad. Según estos resultados, existe una clara mejora en 2020. Respecto al desarrollo de las competencias, cada semestre académico finaliza con un proyecto final con presentación de los estudiantes para la validación de los resultados de aprendizaje. Según los resultados académicos, además de la percepción de los profesores a cargo de los cursos, durante 2020 los estudiantes que aprobaron el curso si logran. En Perú, las calificaciones se miden en una escala de 1 a 20 puntos con un $60 \%$ de exigencia para la aprobar.

Para medir la satisfacción de los estudiantes del periodo académico 2020, se aplicaron encuestas acerca del uso de Arduino para el aprendizaje de programación. A continuación, se presentan parte de la encuesta con 
sus resultados los que resultan una motivación para continuar con esta metodología de enseñanza aprendizaje. Las respuestas son en una escala de Likert de valores entre 1 y 5 , donde 1 representa totalmente en desacuerdo y 5 totalmente de acuerdo:

- Arduino para el aprendizaje de conceptos básicos de programación: 89\% (72\% totalmente de acuerdo y 17\% de acuerdo) le parece que Arduino es una herramienta muy interesante para iniciar en la programación; $76 \%$ ( $41 \%$ totalmente de acuerdo y $35 \%$ de acuerdo) ha logrado comprender el concepto de variable; $72 \%$ ( $41 \%$ totalmente de acuerdo y $31 \%$ de acuerdo) ha aprendido a diferenciar y hacer uso de los operadores lógicos; $62 \%$ ( $41 \%$ totalmente de acuerdo y $21 \%$ de acuerdo) ha logrado comprender el uso de las estructuras condicionales si, sino so, y de lo contrario; $72 \%$ ( $41 \%$ totalmente de acuerdo y $31 \%$ de acuerdo) ha logrado comprender el uso de las estructuras repetitivas para; y $68 \%$ ( $38 \%$ totalmente de acuerdo y $28 \%$ de acuerdo) ha logrado comprender el uso de las estructuras repetitivas mientras.

- Arduino como elemento motivador para continuar estudiándolo: $76 \%$ (14\% totalmente de acuerdo y $62 \%$ de acuerdo) se siente preparado para realizar un proyecto con Arduino; $69 \%$ (28\% totalmente de acuerdo y $41 \%$ de acuerdo) cree que es una plataforma ideal para dar solución a problemas reales; $86 \%$ (55\% totalmente de acuerdo y $31 \%$ de acuerdo) piensa adquirir un kit de Arduino para sus proyectos personales; $87 \%$ (52\% totalmente de acuerdo y $35 \%$ de acuerdo) piensa seguir un curso de Arduino más avanzado. Según estos resultados, es claramente beneficioso el uso de Arduino como herramienta para el desarrollo de competencias de programación, además de desarrollar competencias básicas de electrónica y electricidad.

\section{DISCUSIÓN}

El desarrollo de competencias de programación resulta un tópico de amplio estudio y con diferentes ámbitos de trabajo e investigación en la literatura del mundo. Tsai (2019) menciona la complejidad de aprender lenguajes de programación con estructura sintáctica y semántica complejas, y destaca los efectos positivos del uso de lenguajes de programación visual para el desarrollo de estas competencias. En el contexto de metodologías enseñanza - aprendizaje en cursos de programación, Çakıroğlu et al. (2017) presentan resultados de aplicar una metodología de clase invertida para el desarrollo de competencias de programación. Tal como lo destacan los trabajos (Vidal-Silva et al., 2019; Vidal et al., 2019), en general, varios países han implementado programas para desarrollar habilidades y competencias de programación en educación, y uno de los desafíos inherentes de la enseñanza de la programación es hacerla menos teórica y más atractiva, y el uso de Arduino, por la madurez y nivel académico de estudiantes de estudios superiores, resulta altamente atractivo con resultados y experiencias aplicables en la cotidianeidad. Por ejemplo, con sistemas de sensores y led, los estudiantes están habilitados para medir variables propias en su hogar.

Tabla 1: Resultados estadísticos de rendimiento estudiantil durante 2019 y 2020

\begin{tabular}{|c|c|c|c|}
\hline \multicolumn{2}{|c|}{2019} & \multicolumn{2}{|c|}{2020} \\
\hline promedio & 14.40 & promedio & 15.36 \\
\hline mediana & 15.00 & mediana & 16.00 \\
\hline moda & 14.00 & Moda & 20.00 \\
\hline \multicolumn{2}{|c|}{ Cantidad de estudiantes: 161} & \multicolumn{2}{|c|}{ Cantidad de estudiantes: 128} \\
\hline \multicolumn{2}{|c|}{$2019-1$} & \multicolumn{2}{|c|}{$2020-1$} \\
\hline promedio & 14.97 & Promedio & 16.00 \\
\hline mediana & 16.00 & Mediana & 17.00 \\
\hline moda & 16.00 & Moda & 20.00 \\
\hline \multicolumn{2}{|c|}{ Cantidad de estudiantes: 111} & \multicolumn{2}{|c|}{ Cantidad de estudiantes: 72} \\
\hline \multicolumn{2}{|c|}{$2019-2$} & \multicolumn{2}{|c|}{$2020-2$} \\
\hline promedio & 13.14 & Promedio & 14.54 \\
\hline mediana & 13.00 & Mediana & 14.50 \\
\hline moda & 13.00 & Moda & 14.00 \\
\hline \multicolumn{2}{|c|}{ Cantidad de estudiantes: 50} & \multicolumn{2}{|c|}{ Cantidad de estudiantes: 56} \\
\hline
\end{tabular}


Arduino presenta múltiples beneficios para aplicarlo en entornos educativos. El uso de Arduino en el proceso de enseñanza - aprendizaje, cuando los estudiantes diseñan su sistema, permite mejorar las habilidades de programación entre los estudiantes novatos (Jang et al., 2015). En un estudio sobre el uso de Arduino para la programación de enseñanza en estudiantes de pregrado, Perenc et al. (2019) mostró que el uso de esta herramienta generó un alto compromiso con los estudiantes y mejoró el atractivo del curso. El trabajo de Serrano-Pérez et al. (2018) mostró que la integración de software educativo gratuito y dispositivos electrónicos y mecánicos de bajo costo como herramientas de enseñanza y aprendizaje permiten aumentar la confianza, el rendimiento y el interés en los estudiantes de ingeniería. Finalmente, por el precio asequible y la disponibilidad de Arduino, los estudiantes sin habilidades prácticas o conocimiento en el campo de la electrónica pueden crear aplicaciones simples que están más allá de sus logros en los cursos normales de programación basados en consola (Duch y Jaworski, 2018). A pesar de la importancia futura del desarrollo de habilidades informáticas electrónicas en estudiantes de primaria, los trabajos que abordan este problema no abundan en la literatura. Este artículo desencadena la discusión sobre la enseñanza de herramientas tecnológicas como Scratch y Arduino en establecimientos de educación primaria en Chile.

\section{CONCLUSIONES}

De acuerdo al trabajo presentado y a los resultados obtenidos, se pueden plantear las siguientes conclusiones principales:

1.- Arduino representa una herramienta altamente adecuada para la enseñanza de cursos iniciales de programación gracias a sus propiedades de aprendizaje visual simulado y concreto.

2.- Tinkercad facilita el desarrollo de competencias algorítmicas y de programación gracias a la programación por bloques estilo Scratch y al lenguaje de programación propio de Arduino.

3.- Según los resultados, los estudiantes de las actuales generaciones se sienten muy cómodos con el uso de Arduino y Tinkercad, aun cuando, por el contexto de pandemia, los experimentos no fueron desarrollados de forma física.

\section{REFERENCIAS}

Çakıroğlu, Ü., y Öztürk, M., Flipped classroom with problem-based activities: exploring self-regulated learning in a programming language course, Journal of Educational Technology \& Society, 20(1), 337-349 (2017)

Chorfi, A., Hedjazi, D., Aouag, S., y Boubiche, D., Problem-based collaborative learning groupware to improve computer programming skills, doi: 10.1080/0144929X.2020.1795263, Behaviour \& Information Technology (2020)

Duch, PJ., y Jaworski, T., Enriching computer science programming classes with Arduino game development, https://doi.org/ 10.1109/HSI.2018.8430994, 11 th International Conference on Human System Interaction (HSI), 148-154, Polonia (2018)

González-Guerra, L. H., y Leal-Flores, A. J., Potentializing the problem-solving competence in programming courses through a practice-based learning + tutoring strategy, doi: 10.1109/EDUCON45650.2020.9125154, IEEE Global Engineering Education Conference (EDUCON), Porto, Portugal, 691-697 (2020)

Hooshyar, D., Ahmad, R., y otros 3 autores, Flowchart-based programming environments for improving comprehension and problem-solving skill of novice programmers: a survey, https://10.1504/IJAIP.2015.070343, International Journal of Advanced Intelligence Paradigms, 7(11) (2014)

IICG-Malla, Ingeniería en Información y Control de Gestión - Universidad Católica del Norte, (en línea), https://www.ucn.cl/. Acceso: 04 de Febrero (2021)

Jang, Y., Lee, W., y Kim, J., Assessing the usefulness of object-based programming education using Arduino, doi: 10.17485/ijst/2015/v8iS1/57701, Indian Journal of Science and Technology, 8(S1), 89 (2015)

Juškevičienè, A., Developing algorithmic thinking through computational making, doi: 10.1007/978-3-030-39250-5_10, Data Science: New Issues, Challenges and Applications, Springer International Publishing, 183-197 (2020)

Lineros, M., Bastias, B., y otros 13 autores, Electronics for everybody: student practical experiences using Arduino, doi: 10.1109/SCCC.2018.8705228, 37th International Conference of the Chilean Computer Science Society, Santiago, Chile, IEEE Explore, 1-8 (2018)

Madigan, S., McArthur, B. A., y otros 3 autores, Associations between screen use and child language skills: a systematic review and meta-analysis, doi: 10.1001/jamapediatrics.2020.0327, JAMA pediatrics, 174(7), 665-675, Julio (2020)

Malik, S. I., y Coldwell-Neilson, J., Impact of a new teaching and learning approach in an introductory programming course, https://doi.org/10.1177/0735633116685852, Journal of Educational Computing Research, 5(6), Enero (2017)

Perenc, I., Jaworski, T., y Duch, P., Teaching programming using dedicated Arduino educational board, doi: 10.1002/cae.22134, Computer Applications in Engineering Education, 27(4), 943-954 (2019) 
PSelnt, Una invitación a entrar en el maravilloso mundo de la programación, (en línea), http://pseint.sourceforge.net/. Acceso: 04 de Febrero (2021)

Qian, Y., y Lehman, J., Students' misconceptions and other difficulties in introductory programming: a literature review, https://doi.org/10.1145/3077618, ACM Transactions on Computing Education (TOCE), 18(1), 1-24 (2017)

Resnick, M., Maloney, J., y otros nueve autores, Scratch: programming for all, doi: 10.1145/1592761.1592779, Communications of the ACM, 52(11) 60-67 (2009)

Serrano-Pérez, E. y Juárez-López. F., An ultralow cost line follower robot as educational tool for teaching programming and circuit's foundations, doi: 10.1002/cae.22074, Computer Applications in Engineering Education, 27(2), $288-302$ (2018)

Sirkin, D., Martelaro, N., y Ju, W., Make this! introduction to electronics prototyping using Arduino, https://doi.org/10.1145/3027063.3027094, Actas de CHI Conference Extended Abstracts on Human Factors in Computing Systems, ACM, Nueva York, USA, 1224-1227 (2017)

Tinkercad, Tinkercad - Autodesk Tinkercad - Circuits, (en línea), https://www.tinkercad.com/. Acceso:04 de Febrero (2021)

Troussas, C., Krouska. A., y Sgouropoulou, C., A novel teaching strategy through adaptive learning activities for computer programming, doi: 10.1109/TE.2020.3012744, IEEE Transactions on Education (2020)

Tsai, C. Y., Improving students' understanding of basic programming concepts through visual programming language: The role of self-efficacy, doi: 10.1016/j.chb.2018.11.038, Computers in Human Behavior, 95, 224-232, Junio (2019)

UCON-Malla, Ingeniería de Sistemas e Informática - Universidad Bernardo O’Higgins, (en línea), http://www.ubo.cl/. Acceso: 04 de Febrero (2021)

Velasco-Ramírez, M. L., Resolución de problemas algorítmicos y objetos de aprendizaje: una revisión de la literatura, https://doi.org/10.23913/ride.v10i20.630, RIDE, Revista Iberoamericana para la Investigación y el Desarrollo Educativo, 10(2), Guadalajara, México (2020)

Vidal, C., Cabezas, C., Parra, J., y López, L., Experiencias prácticas con el uso del lenguaje de programación Scratch para desarrollar el pensamiento algorítmico de estudiantes en Chile, Formación Universitaria, 8 (4), 23-32 (2015)

Vidal. C., y Pavesi, L., Desarrollo de un sistema de adquisición y tratamiento de señales electrocardiográficas, Revista Facultad de Ingeniería Universidad de Tarapacá, 13(1), 39-46, Chile (2005)

Vidal-Silva, C., Serrano-Malebran, J., y Pereira, F., Scratch and Arduino for effectively developing programming and computing-electronic competences in primary school children, doi: 10.1109/SCCC49216.2019.8966401, 38th International Conference of the Chilean Computer Science Society (SCCC), Concepcion, Chile, IEEE Explore, 1-7 (2019)

Vidal, C., Lineros, M., Uribe, G., y Olmos, C., Electrónica para todos con el uso de Arduino: experiencias positivas en la implementación de soluciones hardware-software, doi: 10.4067/S0718-07642019000600377, Información Tecnológica, 30(6), 377-386 (2019)

Young, K., Children and technology: guidelines for parents-rules for every age [leading edge], doi: 10.1109/MTS.2017.2670223, IEEE Technology and Society Magazine, 36(1), 27-29 (2017) 
